IOS Press

\title{
A pilot study to investigate bioavailability of strawberry anthocyanins and characterize postprandial plasma polyphenols absorption patterns by Q-TOF LC/MS in humans
}

\author{
Katarzyna Banaszewski, Eunyoung Park, Indika Edirisinghe, Jack C. Cappozzo \\ and Britt M. Burton-Freeman* \\ Center for Nutrition Research, Institute for Food Safety and Health, Illinois Institute of Technology, Bedford Park, \\ IL, USA
}

Received 27 February 2013; accepted 10 April 2013

\begin{abstract}
.
BACKGROUND: Information on absorption and metabolism of strawberry polyphenols is limited.

OBJECTIVE: The aim of this study was to characterize and quantify plasma polyphenols/anthocyanins across 4 doses of strawberry using Q-TOF LC/MS and LC-MS/MS analysis.

METHOD: Plasma was collected from 5 subjects $(n=5)$ every 30-60 min for $6 \mathrm{~h}$ after consuming beverages containing 0,10 , 20 or $40 \mathrm{~g}$ freeze-dried strawberry powder with a meal.

RESULTS: Q-TOF LC/MS and LC-MS/MS analysis of plasma revealed 33 compounds; 7 not reported previously. Pelargonidin$O$-glucuronide (PG) was the most abundant metabolite. Maximum concentrations $\left(\mathrm{C}_{\mathrm{max}}\right)$ of $\mathrm{PG}$ were achieved at $148 \pm 31 \mathrm{~min}$ and were significantly different among beverages containing $0,10,20,40 \mathrm{~g}$ strawberry powder: $0,93.4 \pm 21.9,166.5 \pm 16.2$ and $226.7 \pm 36.7 \mathrm{nmol} / \mathrm{L}$, respectively $(P<0.05)$. Area under the concentration curve (AUC) also increased with dose $(P<0.05)$; however, $\mathrm{C}_{\max }$ and $\mathrm{AUC}$ of $\mathrm{PG}$ was reduced as a percent of pelargonidin-3-O-glucoside $(\mathrm{P} 3 \mathrm{G})$ delivered in the strawberry beverages $(P<0.05)$.

CONCLUSION: Use of both Q-TOF LC/MS and LC-MS/MS allowed for detection of compounds in plasma not previously reported, which may be a useful approach for characterizing postprandial plasma metabolites of lesser known plant foods. Additionally, higher concentrations of key strawberry compounds/metabolites are achieved with eating more strawberry; however, saturation of absorptive capacity of pelargonidin-based anthocyanins was suggested.
\end{abstract}

Keywords: Bioavailability, anthocyanin, Q-TOF LC/MS, LC-MS/MS, strawberry

\section{Introduction}

Interest in berry fruits for their beneficial health effects has increased dramatically in recent years. Strawberries, blueberries, blackberries and raspberries have received the most attention due to their content and composition of bioactive compounds such as anthocyanins, catechins and flavanols [1-3]. Studies conducted both in vitro and

*Corresponding author: Britt M. Burton-Freeman, PhD, MS, Institute for Food Safety and Health, Illinois Institute of Technology, Moffett Campus, 6502 S. Archer Road, Bedford Park, IL 60501, USA. Tel.: +1 708341 7078; Fax: +1 708563 1873; E-mail: bburton@iit.edu. 
in vivo using berries (as whole fruit) or their phytochemical constituents suggest an important role in reducing the risk of cancer [4, 5], atherosclerosis [6] diabetes [7-9], age-related memory decline [10] and more [11].

Specifically, consumption of strawberries by humans in doses ranging from $\sim 100 \mathrm{~g}$ to $500 \mathrm{~g}$ fresh weight have been shown to decrease fasting lipid concentrations and attenuate postprandial oxidative- and inflammatory- stress concurrent with improved postprandial insulin action [12-16]. Presumably, much of the documented effects of strawberries are owed to the antioxidant and cell signaling properties of the polyphenolic, particularly anthocyanin, compounds they provide when consumed. Strawberries are unique in that $\sim 98$ percent of their total anthocyanin content is derived from pelargonidin-based anthocyanins with pelargonidin-3- $O$-glucoside providing the greatest contribution; $\sim 84$ percent [15], which is among other things, cultivar- dependent. Pelargonidin-3-O-glucoside is considered one of the most bioavailable anthocyanins and can be found in plasma and urine in its native form or as methylated, glucuronidated or sulfated conjugates [16-18]. Pelargonidin- $O$-glucuronide is the predominant plasma metabolite associated with strawberry consumption with measurable amounts of pelargonidin-sulfate [16-18]. However, in general, studies examining bioavailability of anthocyanins indicate that they are poorly absorbed and, in the case of some anthocyanins, thresholds for maximum absorption may be apparent $[17,19,20]$. Since it is generally assumed that to impart benefit, nutrients/bioactive components must be absorbed, factors that influence their bioavailability and the metabolites they bestow are of interest. Recent studies examining the bioavailability of pelargonidin and other anthocyanins of strawberry suggest that storage, dose and dietary fat can impact absorption kinetics and metabolite concentrations [18, 21-23]. However, no studies that we are aware of have examined strawberry consumed over multiple doses in a typical meal setting, in a beverage with a complete meal. Because meals, and particularly western style meals, are pro-oxidative and pro-inflammatory, it is of interest to determine the relative bioavailability and absorption kinetics of strawberry bioactives in a situation where the availability of the fruit components will be important for attenuating unfavorable meal-induced responses. Therefore, the primary focus of the present study was to characterize the plasma profile of strawberry anthocyanins, particularly the pelargonidin-based anthocyanins across 4 doses during the postprandial phase of metabolism; and secondly, to investigate the utility of employing targeted and non-targeted analysis approaches to advance our understanding of secondary-plant metabolite handling in vivo.

\section{Methods}

\subsection{Subjects}

The investigation involved human subjects and was conducted according to the guidelines laid down in the Declaration of Helsinki. All procedures involving human subjects were approved by the local Institutional Review Board, which protects the rights and welfare of human subjects in research studies conducted under the auspices of the Illinois Institute of Technology, IL, USA. All subjects provided written informed consent prior to any study related procedures.

Five subjects participated in this pilot study. Subjects were recruited from the Chicago, IL, USA community and met basic inclusion/exclusion exclusion criteria including: 18 years or older, non-smokers, and have no allergies or intolerances to strawberries or other study foods. Individuals with diabetes mellitus or other metabolic/systemic diseases/disorders or used medications or dietary supplements that would interfere with study endpoints were not eligible to participate. Participants were obese [body mass index (BMI) of 42.1 \pm 9.7 ] men $(n=1)$ and women $(n=4)$.

\subsection{Study design, treatments and procedures}

This was a single-center, randomized, single-blind, four-arm, cross-over trial conducted at the Clinical Nutrition Research Center (CNRC) at the Institute of Food Safety and Health, Illinois Institute of Technology in Chicago, Illinois. The basic design involved the administration of a test meal with treatment beverages followed by periodic blood sampling. Eligible subjects were required to avoid consuming berries, including strawberries, while maintaining all other aspects of their diet and physical activity for the duration of the experiment, starting 7 days before the first study day session. During the experimental period, subjects consumed four test meals in random order on four different 
Table 1

Test meal for postprandial study days

\begin{tabular}{lc}
\hline Food $^{1}$ & Amount $(\mathrm{g})$ \\
\hline Bagel & 110.0 \\
Cream Cheese & 14.0 \\
Margarine & 5.0 \\
Hard-boiled Egg & 50.0 \\
Cantaloupe & 85.0 \\
Whole Milk & 240.0 \\
\hline
\end{tabular}

${ }^{1}$ All foods were purchased from local Jewel stores in Chicago, IL and prepared fresh on each study day (10-30 min prior to breakfast time).

Table 2

Ingredients of the placebo and strawberry beverages

\begin{tabular}{|c|c|c|c|c|}
\hline Ingredients & $\begin{array}{c}\text { Placebo } \\
\text { beverage }\left(0 \text { g powder }^{1}\right)\end{array}$ & $\begin{array}{c}\text { Strawberry } \\
\text { beverage ( } 10 \mathrm{~g} \text { powder })\end{array}$ & $\begin{array}{c}\text { Strawberry } \\
\text { beverage ( } 20 \mathrm{~g} \text { powder })\end{array}$ & $\begin{array}{c}\text { Strawberry } \\
\text { beverage ( } 40 \mathrm{~g} \text { powder) }\end{array}$ \\
\hline Freeze Dried strawberry powder ${ }^{1}(\mathrm{~g})$ & 0 & 10 & 20 & 40 \\
\hline Strawberry Nesquick powder ${ }^{2}(\mathrm{~g})$ & 48 & 30 & 28 & 9 \\
\hline Skim milk powder ${ }^{3}(\mathrm{~g})$ & 22 & 19 & 18 & 18 \\
\hline Evaporated milk ${ }^{4}(\mathrm{~g})$ & 25 & 15 & 10 & 3 \\
\hline Water (g) & 237 & 237 & 237 & 237 \\
\hline Whole milk ${ }^{5}(\mathrm{~g})$ & 240 & 240 & 240 & 240 \\
\hline White granulated sugar ${ }^{6}(\mathrm{~g})$ & 4.5 & 18 & 15 & 20 \\
\hline Unifiber $^{7}(\mathrm{~g})$ & 11.3 & 8.5 & 5.7 & 0 \\
\hline Vitamin $\mathrm{C}^{8}(\mathrm{mg})$ & 110.9 & 105.5 & 93.7 & 75.4 \\
\hline Total (g) & 348.1 & 337.6 & 333.1 & 327.1 \\
\hline
\end{tabular}

${ }^{1}$ Freeze-dried strawberry powder, California Strawberry Commission (Watsonville, CA, USA). ${ }^{2}$ Nesquik strawberry artificially flavored powder (Nestle' USA, Inc., Glendale, CA, USA). ${ }^{3}$ Premium Sanalac Nonfat Dry Milk (ConAgra Foods, Inc., Omaha, NE, USA). ${ }^{4}$ Evaporated milkNestlé brand: Carnation: Vitamin D added. ${ }^{5}$ Whole milk - Dean's Milk, Vitamin D added. ${ }^{6}$ Domino sugar (Domino Foods, Yonkers, NY, USA). ${ }^{7}$ Unifiber - DrNatura Unifiber, Natural Fiber Supplement, 8.4-Ounce. ${ }^{8}$ Food Graded Vitamin C: Brand (Nature's Flavors), Ascorbic Acid; $100 \%$ pure Food grade, gluten free, and vegan.

occasions so that each subject served as his/her own control. Test meals consisted of breakfast food items (Table 1), which were accompanied by 1 of 4 beverages (Table 2) containing $0,10,20$ or $40 \mathrm{~g}$ freeze-dried strawberry powder (California Strawberry Commission, Watsonville, CA, USA- The detail polyphenolic composition of California strawberry powder is given in Table 5- ref 15). The composition of beverages was closely matched in terms of total energy, macronutrients, vitamin $\mathrm{C}$ and fiber content (Table 3).

All beverages were prepared fresh in the metabolic kitchen at the CNRC. Beverage ingredients were weighed on a scale to $0.1 \mathrm{~g}$. All ingredients except for the freeze-dried strawberry powder were mixed in a blender until smooth. The freeze-dried strawberry powder was sprinkled into the smooth mixture while continuing to blend. After transferring the beverage to a cup, the blender was rinsed and the rinse water was added to the smoothie beverage. Following consumption of the beverage, the cup was also rinsed with water, which was then consumed by the subject to maximize the accuracy of anthocyanin intake. Beverages were served with meals in non-transparent cups with opaque lids and straws.

The postprandial test was conducted according to a standardized protocol [15]. Briefly, the subjects reported to the laboratory in the morning in a fasting state on four occasions 3-5 d apart. Indwelling catheters were placed in the antecubital vein of the non-dominant arm of the subjects, and blood samples were collected to EDTA tubes before (time $0 \mathrm{~min}$ ) and at multiple time points after the meal, 30, 60, 90, $120 \mathrm{~min}$, and then hourly, thereafter, to $6 \mathrm{~h}$. EDTA 
Table 3

Test meal and beverage nutrient composition, including anthocyanin content

\begin{tabular}{|c|c|c|c|c|}
\hline Nutrient & $\begin{array}{c}\text { Meal }+ \text { placebo } \\
\text { beverage }\left(0 \text { g powder }^{1}\right)\end{array}$ & $\begin{array}{c}\text { Meal + strawberry } \\
\text { beverage ( } 10 \mathrm{~g} \text { powder })\end{array}$ & $\begin{array}{c}\text { Meal + strawberry } \\
\text { beverage ( } 20 \mathrm{~g} \text { powder })\end{array}$ & $\begin{array}{c}\text { Meal + strawberry } \\
\text { beverage ( } 40 \mathrm{~g} \text { powder) }\end{array}$ \\
\hline Energy (Kcal) & 963.7 & 951.1 & 949.3 & 971.6 \\
\hline Energy from fat (Kcal) & 223.1 & 221.4 & 222.6 & 228.0 \\
\hline Carbohydrate (g) & 146.4 & 146.9 & 147.4 & 152.9 \\
\hline Total Sugar (g) & 90.3 & 88.8 & 87.4 & 88.2 \\
\hline Dietary Fiber (g) & 12.3 & 12.1 & 12.0 & 12.5 \\
\hline Protein $(g)$ & 37.4 & 36.1 & 36.0 & 37.5 \\
\hline Fat $(\mathrm{g})$ & 24.8 & 24.6 & 24.7 & 25.3 \\
\hline Anthocyanins ( $\mu \mathrm{mol})$ & $0.1 \pm 0.0$ & $100.9 \pm 2.7$ & $210.3 \pm 5.7$ & $368.8 \pm 12.1$ \\
\hline Pelargonidin-3-O-glucoside ( $\mu \mathrm{mol})$ & 0 & $81.3 \pm 1.8$ & $166.8 \pm 4.5$ & $285.8 \pm 8.9$ \\
\hline
\end{tabular}

${ }^{1}$ Powder $=$ Freeze-dried Strawberry Powder (California Strawberry Commission, Watsonville, CA).

plasma was stored at $-80^{\circ} \mathrm{C}$ until used for analysis. The meal and the beverages were consumed entirely under supervision in $20 \mathrm{~min}$.

\subsection{Chemicals}

Pelargonidin-3-O-glucoside, pelargonidin-chloride, cyanidin-3-O-glucoside, (+)-catechin, ellagic acid, isoquercetin, kaempferol, quercetin-3-O-glucoside and kaempferol-3- $O$-rutinoside were purchased from Chromadex Inc. (Santa Ana, CA). All solvents were HPLC-MS grade and were purchased from Fisher Scientific (Pittsburg, PA).

\subsection{Plasma and beverage sample preparation}

Prior to extraction, $50 \mu \mathrm{L}$ of delphinidin-3-O-glucoside $(1 \mu \mathrm{g} / \mathrm{mL})$ was added to $500 \mu \mathrm{L}$ of plasma samples as an internal standard. Samples were acidified to $\mathrm{pH} 2$ with formic acid (FA) and extracted with $1.5 \mathrm{~mL}$ of acetonitrile $(\mathrm{ACN})$, centrifuged at $10,000 \mathrm{rpm}$ for $15 \mathrm{~min}$ at $4^{\circ} \mathrm{C}$ then re-extracted with $1.5 \mathrm{~mL}$ methanol. Extracts were pooled, evaporated under nitrogen at $35^{\circ} \mathrm{C}$, reconstituted in $250 \mu \mathrm{L}$ of mobile phase and centrifuged at $10,000 \mathrm{rpm}$ for 30 min at $4^{\circ} \mathrm{C}$. Samples were transferred to amber HPLC vials and immediately analyzed by Q-TOF LC/MS and by LC-MS/MS.

Beverages containing $0,10,20$, or $40 \mathrm{~g}$ freeze dried strawberry powder were also prepared for analysis. In brief, a triplicate of each $1 \mathrm{~mL}$ sample was extracted with $15 \mathrm{~mL}$ of acetone:water (70:30, v/v) by shaking for $20 \mathrm{~min}$. Samples were then centrifuged at $10,000 \mathrm{rpm}$ and the clear extract was dried under nitrogen at $35^{\circ} \mathrm{C}$. Dried pellets were then dissolved in $2 \mathrm{~mL}$ of mobile phase filtered through a $0.2 \mu \mathrm{m}$ PTFE syringe filter (Phenomenex, Torrance, CA) and submitted for LC-MS/MS analysis. Table 4 shows a list of polyphenolic compounds detected in the strawberry-based beverages by LC-MS/MS.

\subsection{LC-MS/MS and Q-TOF LC/MS analysis}

Sample analysis was performed on an Agilent Technologies 1260 Infinity HPLC system with a 6460 Triple Quadruple Mass Spectrometer (Agilent Technologies, Santa Clara, CA) and a 1290 Infinity HPLC system (Agilent Technologies) coupled with an Agilent 6530 Accurate-Mass Q-TOF LC/MS system. Samples were placed in cooled autosamplers $\left(4^{\circ} \mathrm{C}\right)$ to maintain sample stability throughout the analysis.

Chromatographic separation on both systems was achieved on Agilent Poroshell $120 \mathrm{SB}-\mathrm{C} 183.0 \times 150 \mathrm{~mm}$, $2.7 \mu \mathrm{m}$ columns (Agilent Technologies). The mobile phase was a gradient of $0.2 \% \mathrm{FA}$ in water (A) and ACN (B) at a flow rate of $0.3 \mathrm{ml} / \mathrm{min}$. The gradient starting composition was $2 \% \mathrm{~B}$ increasing to $100 \% \mathrm{~B}$ over 15 min followed by a 4 min post-run. For both instruments detection was carried out by using electrospray ionization (ESI) with nebulizer 
Table 4

Qualitative analysis of polyphenolic compounds in strawberry-based beverage

\begin{tabular}{|c|c|c|c|c|}
\hline Compound & Ion $(+/-)$ & Retention time & $\mathrm{MS} \mathrm{m} / \mathrm{z}$ & $\mathrm{MS}^{2} \mathrm{~m} / \mathrm{z}$ \\
\hline Hydroxybenzoylhexose & - & 4.0 & 299 & $239,179,137$ \\
\hline bis-HHDP-glucose & - & 4.8 & 783 & $481,301,257,229$ \\
\hline Proanthocyanidin trimer & - & 5.1 & 865 & 739,695 \\
\hline p-Coumaroylhexose & - & 5.2 & 325 & 163,119 \\
\hline p-Coumaroylhexose-4-O-hexoside & - & 5.6 & 487 & $325,163,119$ \\
\hline Proanthocyanidin B1 & - & 5.7 & 577 & 425,407 \\
\hline Proanthocyanidin dimer & - & 5.7 & 577 & 407,289 \\
\hline Cyanidin-diglucoside & + & 5.9 & 611 & 449,287 \\
\hline Cyanidin-3-O-glucoside & + & 5.9 & 449 & 287 \\
\hline Galloyl-HHDP-glucose & - & 6 & 633 & 481,301 \\
\hline Kaempferol-3-coumaroylglucoside & - & 6 & 593 & 447,285 \\
\hline$(+)$-Catechin & - & 6.3 & 289 & 245 \\
\hline Pelargonidin-3-O-glucoside & + & 6.6 & 433 & 271,121 \\
\hline Pelargonidin-3-O-rutinoside & + & 6.7 & 579 & 433,271 \\
\hline Pelargonidin & + & 6.7 & 271 & 121 \\
\hline Pelargonidin-3-malonyl-glucoside & + & 8.1 & 519 & 433,271 \\
\hline Quercetin-3-O-rutinoside & - & 8.2 & 609 & $301,151,179$ \\
\hline Galloyl-bis-HHDP-glucoside & - & 8.4 & 935 & 633,301 \\
\hline Quercetin-3-O-glucoside & - & 8.7 & 463 & $301,151,179$ \\
\hline Quercetin-3-O-galactoside & - & 8.7 & 477 & $301,151,179$ \\
\hline Ellagic acid (EA) & - & 8.7 & 301 & $257,229,185$ \\
\hline Kaempferol-glucuronide & - & 9.5 & 461 & $285,179,161$ \\
\hline Kaempferol-3-malonylglucoside & - & 10.3 & 533 & 285 \\
\hline Pelargonidin-3-malonyl-rhamnoside & + & 10.8 & 503 & 271 \\
\hline
\end{tabular}

pressure of $35 \mathrm{psi}$, drying nitrogen gas flow of $10 \mathrm{~L} / \mathrm{min}$ at $325^{\circ} \mathrm{C}$, and sheath gas flow of $11 \mathrm{~L} / \mathrm{min}$ at $350^{\circ} \mathrm{C}$. Capillary voltage was set to $3500 \mathrm{~V}$ and nozzle voltage to $500 \mathrm{~V}$. Acquisition type for Q-TOF was Auto MS/MS (data-dependent) in High Resolution $(4 \mathrm{GHz})$ mode at a rate of $2 \mathrm{spectra} / \mathrm{sec}$ in positive and negative ion mode. Collision energies were 16 and $28 \mathrm{~V}$ and charge state was $1+$ and unknown. A differential analysis of the Q-TOF data were performed using Mass Profiler software (Agilent Technologies). Compounds were identified based on their accurate mass and $\mathrm{MS}^{2}$ fragmentation pattern of parent ion. The Molecular Feature Extractor (MFE) algorithm in the MassHunter Qualitative analysis software (Agilent Technologies) was used to determine possible metabolites present in plasma samples. In short, the MFE maps signals in the 3-dimentional space in time and mass at the MS level, then removes noise areas with no signals, identifies all mass signals with a common retention time (RT), combines mass signals with common RT and chemical relation (isotope, adduct, dimer, higher charge state) and creates Extracted Compound Chromatograms (ECC) and Compound Mass Spectra from ions associated to a molecular feature (compound). Where available, compound identity was confirmed by hits from existing databases [Metlin - Scripps, PCDL (MassHunter Personal Compound Database and Library - Agilent Technologies)].

In the LC-MS/MS analysis, anthocyanins were detected in positive $[\mathrm{M}-\mathrm{H}]^{+}$, and all other compounds in negative $[\mathrm{M}-\mathrm{H}]^{-}$, ionization mode utilizing fast polarity switching feature. Since there is a resistance towards using fast polarity switching within one run due to a potential sensitivity loss, an analysis was performed to validate the fast polarity switching against standard methods utilizing either positive or negative ion mode (data not shown). No significant reduction of sensitivity was observed; therefore the positive and negative ion mode methods were combined. The LC-MS/MS analysis was carried out using Multiple Reaction Monitoring (MRM) by monitoring specific transitions of parent and product ions. Compounds were quantified using reference standards where available. Otherwise, relative quantitation was applied. Matrix-matched pelargonidin-3-O-glucoside standard curve (range $0.1-1000 \mathrm{ng} / \mathrm{mL}$ ) was used to relatively quantify all pelargonidin-based compounds. 


\subsection{Calculations and statistical analysis}

Maximum plasma concentrations $\left(\mathrm{C}_{\max }, \mathrm{nmol} / \mathrm{L}\right)$ were determined based on the highest concentration of metabolites measured from 0 to $6 \mathrm{~h}$ post dose. The time to maximum plasma concentrations $\left(\mathrm{T}_{\max }\right)$ was defined as the time in minutes $(\mathrm{min})$ at which $\mathrm{C}_{\max }$ was achieved. Incremental area under the $6 \mathrm{~h}$ concentration curves (AUC, $\mathrm{nmol} / \mathrm{L} * \mathrm{~h})$ was calculated using the linear Trapezoidal Method. Elimination rate constant $\left(\mathrm{k}_{\mathrm{e}}{ }^{\mathrm{h}-1}{ }^{1}\right)$ was calculated from the following equation based on data collected during the elimination phase of the time-concentration curves: $\mathrm{k}_{\mathrm{e}}=-\left[\ln \left(\mathrm{C}_{1}\right)-\ln \left(\mathrm{C}_{2}\right)\right] / \mathrm{t}_{1}-\mathrm{t}_{2}$, where $\mathrm{C}=$ concentration and $\mathrm{t}$ is time. Relative bioavailability was calculated based on pelargonidin- $O$-glucuronide $\mathrm{AUC}_{0-6}$ h corrected for individual plasma volume based on sex and body weight (kg) according to the methods described in the technical manual of American Association of Blood Banks (AABB) [24], where plasma volume is estimated to be $36 \mathrm{~mL} / \mathrm{kg}$ for women and $40 \mathrm{~mL} / \mathrm{kg}$ for men. Percent recovery/bioavailability was calculated by dividing the plasma mass (nmol) by the mass of pelargonidin-3-O-glucoside per dose ( $\mu \mathrm{mol})$ and multiplying by 100 .

Statistical analysis was performed using Sigma Plot statistical program version 11.2 (Systat Software Inc. San Jose, CA) and SAS (version 9.1.3; SAS Institute Inc, Cary, NC). All data were tested for normality and equal variance prior to analysis by one way analysis of variance (ANOVA) using Sigma Plot and repeated measures (RM-ANOVA) using MIXED procedure of SAS. Statistical significance was considered with $P$ values $<0.05$. Results are given as the mean \pm SEM or mean \pm SD as indicated, $n=5$. Post hoc power analysis indicated that sufficient power $(>80 \%)$ was provided with the sample size $(n=5)$ on plasma outcome variables reported.

\section{Results}

\subsection{Treatment beverages}

Table 4 shows the qualitative analysis of strawberry polyphenols in strawberry beverages by LC-MS/MS. Quantitative analysis of anthocyanins indicated that the four doses of strawberry powder $(0,10,20,40 \mathrm{~g})$ in beverages delivered $0.1 \pm 0.0,100.9 \pm 2.7,210.3 \pm 5.7$ and $368.8 \pm 12.1 \mu$ mol of anthocyanins of which pelargonidin-3-O-glucoside was $0,81.3 \pm 1.8,166.8 \pm 4.5$ and $285.8 \pm 8.9 \mu \mathrm{mol}$, respectively. The total pelargonidin-based anthocyanin content per dose was $0,97.1 \pm 1.9,200.4 \pm 6.2$ and $346.6 \pm 10.1 \mu \mathrm{mol}$, respectively.

\subsection{Q-TOF LC/MS analysis of polyphenols in plasma}

The analysis of polyphenolic compounds in plasma using Q-TOF LC/MS consisted of three key steps. First, the acquired data were subjected to an algorithm called Molecular Feature Extractor (MFE) which resulted in determination of over 600 compounds present in human plasma. The second step was importing the initially processed data into Mass Profiler (MP) program to perform differential analysis of plasma samples with regard to treatment, time, and subjects. Once the analysis was completed, a database search was performed to identify specified compounds.

Qualitative Q-TOF LC/MS analysis of human plasma after strawberry consumption revealed 33 phenolic compounds and their metabolites (Table 5). Of these compounds, 26 have been reported previously in studies on strawberry polyphenols $[1-3,18,25,26]$ and 7 have not been mentioned before as metabolites recovered in human plasma. Fisetin aglycone (mass 286.0469 Da) and its glycosylated form - fisetin-3rutinoside (mass 594.1583 Da), have not been reported in any of the human studies on strawberry polyphenols, although fisetin is known to be a flavonoid naturally occurring in strawberry fruit [9, 27]. Similarly three additional pelargonidin metabolites were identified in plasma: pelargonidin-3- $O-6^{\prime \prime}$-rhamnosylglucoside (mass $579.1503 \mathrm{Da}), 5$-carboxypyranopelargonidin-3-O-glucopyranoside (mass $501.1034 \mathrm{Da}$ ) and pelargonidin-3-(6" caffeoylglucoside) (mass 595.1461 Da). Quercetin-3-(6"' -caffeoylgalactoside) at mass 626.1213 Da, quercetin-3-(2", $6^{\prime \prime}$-digalloylgalactoside) at mass $768.1174 \mathrm{Da}$ and kaempferol-3-(6"' -caffeoylglucosyl)-(1->2)-galactoside (mass $772.1850 \mathrm{Da}$ ) were also detected in post prandial plasma. The selectivity offered by accurate mass Q-TOF analysis with MS fragmentation allowed detection of trace metabolites, which permitted their identification and assessment of their relative appearance in plasma based on the sampling procedure. 
Table 5

Phenolic compounds determined by Q-TOF LC/MS in plasma ${ }^{1}$

\begin{tabular}{|c|c|c|c|c|c|}
\hline $\mathrm{t}_{\mathrm{R}}(\min )$ & Compound & Estimated mass $(\mathrm{Da})$ & Actual mass (Da) & Error $(\mathrm{ppm})$ & Formula \\
\hline 2.6 & p-Coumaric acid & 164.0473 & 164.0470 & -1.829 & $\mathrm{C}_{9} \mathrm{H}_{8} \mathrm{O}_{3}$ \\
\hline 3.6 & 3,4-Dihydroxybenzoic acid & 154.0266 & 154.0264 & 1.298 & $\mathrm{C}_{7} \mathrm{H}_{6} \mathrm{O}_{4}$ \\
\hline 3.8 & Kaempferol-glucuronide & 462.0798 & 462.0770 & -6.492 & $\mathrm{C}_{21} \mathrm{H}_{18} \mathrm{O}_{12}$ \\
\hline 4.9 & 4-Hydroxybenzoic acid & 138.0320 & 138.0325 & 3.622 & $\mathrm{C}_{7} \mathrm{H}_{6} \mathrm{O}_{3}$ \\
\hline 6.4 & Syringic acid & 198.0528 & 198.05185 & -10.098 & $\mathrm{C}_{9} \mathrm{H}_{10} \mathrm{O}_{5}$ \\
\hline 7.3 & Kaempferol-3-glucoside & 448.1006 & 448.1037 & 7.007 & $\mathrm{C}_{21} \mathrm{H}_{20} \mathrm{O}_{11}$ \\
\hline 7.4 & Pelargonidin-3,5-diglucoside & 595.1663 & 595.1636 & -3.528 & $\mathrm{C}_{27} \mathrm{H}_{31} \mathrm{O}_{15}$ \\
\hline 7.5 & Pelargonidin-3-glucuronide & 447.0927 & 447.094 & 1.924 & $\mathrm{C}_{21} \mathrm{H}_{19} \mathrm{O}_{11}$ \\
\hline 8.1 & Cyanidin-3,5-diglucoside & 611.1598 & 611.1612 & 2.291 & $\mathrm{C}_{27} \mathrm{H}_{31} \mathrm{O}_{16}$ \\
\hline 8.2 & Quercetin-3-glucoside & 464.0955 & 464.0962 & 1.551 & $\mathrm{C}_{21} \mathrm{H}_{20} \mathrm{O}_{12}$ \\
\hline 8.2 & Quercetin-3-galactoside & 464.0955 & 464.0932 & -4.913 & $\mathrm{C}_{21} \mathrm{H}_{20} \mathrm{O}_{12}$ \\
\hline 8.7 & Cyanidin-3-O-glucoside & 449.1084 & 449.1086 & 0.468 & $\mathrm{C}_{21} \mathrm{H}_{21} \mathrm{O}_{11}$ \\
\hline 8.9 & Pelargonidin-3-sambubioside & 565.1557 & 565.1563 & 1.009 & $\mathrm{C}_{26} \mathrm{H}_{29} \mathrm{O}_{14}$ \\
\hline 8.9 & $(+)$ Catechin & 290.0790 & 290.081 & -6.895 & $\mathrm{C}_{15} \mathrm{H}_{14} \mathrm{O}_{6}$ \\
\hline 9.2 & Pelargonidin-3- $O$-glucoside & 433.1135 & 433.1134 & -0.162 & $\mathrm{C}_{21} \mathrm{H}_{21} \mathrm{O}_{10}$ \\
\hline 10.3 & Fisetin & 286.0477 & 286.0469 & 2.796 & $\mathrm{C}_{15} \mathrm{H}_{10} \mathrm{O}_{6}$ \\
\hline 11.6 & Pelargonidin-sulfate & 351.0175 & 351.0136 & -10.997 & $\mathrm{C}_{15} \mathrm{H}_{11} \mathrm{O}_{5} \mathrm{~S}$ \\
\hline 11.7 & Quercetin-rutinoside & 610.1534 & 610.1541 & 1.180 & $\mathrm{C}_{27} \mathrm{H}_{30} \mathrm{O}_{16}$ \\
\hline 11.8 & Quercetin & 302.0426 & 302.0411 & -4.966 & $\mathrm{C}_{15} \mathrm{H}_{10} \mathrm{O}_{7}$ \\
\hline 12.0 & Pelargonidin-3-(6"'-malonylglucoside) & 519.1502 & 519.1531 & -1.926 & $\mathrm{C}_{24} \mathrm{H}_{23} \mathrm{O}_{13}$ \\
\hline 12.1 & Pelargonidin-3-O-6"-rhamnosylglucoside & 579.1503 & 579.1503 & 0.086 & $\mathrm{C}_{30} \mathrm{H}_{27} \mathrm{O}_{12}$ \\
\hline 12.4 & Quercetin-glucuronide & 478.0747 & 478.0766 & 3.891 & $\mathrm{C}_{21} \mathrm{H}_{18} \mathrm{O}_{13}$ \\
\hline 12.5 & Quercetin-sulfate & 381.9994 & 381.9960 & -8.900 & $\mathrm{C}_{15} \mathrm{H}_{10} \mathrm{O}_{10} \mathrm{~S}$ \\
\hline 13.5 & 5-Carboxypyranopelargonidin-3-O-beta-glucopyranoside & 501.1033 & 501.1034 & 0.200 & $\mathrm{C}_{24} \mathrm{H}_{21} \mathrm{O}_{12}$ \\
\hline 15.4 & Kaempferol-3-O-sulfate & 366.0045 & 366.0021 & -0.655 & $\mathrm{C}_{15} \mathrm{H}_{10} \mathrm{O}_{9} \mathrm{~S}$ \\
\hline 17.6 & Kaempferol-3-coumaroylglucoside & 594.1373 & 594.1371 & -0.337 & $\mathrm{C}_{30} \mathrm{H}_{26} \mathrm{O}_{13}$ \\
\hline 19.7 & Fisetin-3-rutinoside & 594.1585 & 594.1583 & -0.337 & $\mathrm{C}_{27} \mathrm{H}_{30} \mathrm{O}_{15}$ \\
\hline 19.9 & Methylquercetin & 316.0583 & 316.0561 & -6.961 & $\mathrm{C}_{16} \mathrm{H}_{12} \mathrm{O}_{7}$ \\
\hline 20.0 & Kaempferol-3-(6"-malonylglucoside $)$ & 534.1010 & 534.1011 & -0.187 & $\mathrm{C}_{24} \mathrm{H}_{22} \mathrm{O}_{14}$ \\
\hline 20.1 & Quercetin-3-(6"'-caffeylgalactoside $)$ & 626.1272 & 626.1273 & 0.159 & $\mathrm{C}_{30} \mathrm{H}_{26} \mathrm{O}_{15}$ \\
\hline 20.6 & Kaempferol 3-(6"' -caffeoylglucosyl)-(1->2)-galactoside & 772.1850 & 772.1852 & 0.155 & $\mathrm{C}_{36} \mathrm{H}_{36} \mathrm{O}_{19}$ \\
\hline 21.2 & Quercetin 3-(2", $6^{\prime \prime}$-digalloylgalactoside $)$ & 768.1174 & 768.1174 & 0.013 & $\mathrm{C}_{35} \mathrm{H}_{28} \mathrm{O}_{20}$ \\
\hline 21.3 & Pelargonidin3-(6" -caffeoylglucoside $)$ & 595.1452 & 595.1461 & 1.512 & $\mathrm{C}_{30} \mathrm{H}_{27} \mathrm{O}_{13}$ \\
\hline
\end{tabular}

${ }^{1}$ Compounds determined based on differences captured using Mass Profiler software, database hits, fragmentation patterns and structure elucidation. $N=5$.

Time of appearance based on the sampling protocol of identified compounds/metabolites is shown in Table 6 . After consumption of the placebo beverage, only 4-hydroxybenzoic acid (reported also by Azzini et al. [18] in the baseline and strawberry postprandial plasma) and quercetin-3-glucoside were detectable in all 5 subjects 30 min post prandial. However, p-coumaric acid and quercetin were also detected at $30 \mathrm{~min}$ postprandial in two subjects. Consumption of the test beverages containing 10, 20 and $40 \mathrm{~g}$ of strawberry powder resulted in the appearance of 8 , 10 and 13 compounds, respectively at $30 \mathrm{~min}$. Between 60 and $180 \mathrm{~min}, \sim 22$ out of 33 compounds were detectable. Some compounds were detected late in the postprandial period, for instance quercetin-3-(6"-caffeylgalactoside), quercetin-rutinoside, 5-carboxypyranopelargonidin-3- $O$-beta-glucopyranoside, kaempferol 3-(6"' -caffeylglucosyl)(1->2)-galactoside were first detectable at approximately 180 minutes; however, for the latter 3 compounds, as dose increased appearance tended to be earlier in most subjects. 
Table 6

Phenolic compounds in plasma based on time (min) of first appearance determined by Q-TOF MS ${ }^{1}$

\begin{tabular}{|c|c|c|c|c|}
\hline \multirow[t]{2}{*}{ Compound } & \multicolumn{4}{|c|}{ Time of first appearance (min) } \\
\hline & $0 \mathrm{~g}$ & $10 \mathrm{~g}$ & $20 \mathrm{~g}$ & $40 \mathrm{~g}$ \\
\hline p-Coumaric acid & $30 \pm 0^{2}$ & $30 \pm 0$ & $30 \pm 0$ & $30 \pm 0$ \\
\hline 3,4-Dihydroxybenzoic acid & ND & $60 \pm 0$ & $60 \pm 0$ & $60 \pm 0$ \\
\hline Kaempferol-glucuronide & ND & $78 \pm 16$ & $66 \pm 13$ & $60 \pm 0$ \\
\hline 4-Hydroxybenzoic acid & $30 \pm 0$ & $48 \pm 16$ & $48 \pm 16$ & $48 \pm 16$ \\
\hline Syringic acid & ND & $60 \pm 0$ & $30 \pm 0$ & $30 \pm 0$ \\
\hline Kaempferol-3-glucoside & ND & $36 \pm 13$ & $30 \pm 0$ & $30 \pm 0$ \\
\hline Pelargonidin-3,5-diglucoside & ND & $30 \pm 0$ & $48 \pm 16$ & $30 \pm 0$ \\
\hline Pelargonidin- $O$-glucuronide & ND & $30 \pm 0$ & $30 \pm 0$ & $30 \pm 0$ \\
\hline Cyanidin-3,5-diglucoside & ND & $54 \pm 13$ & $30 \pm 0$ & $30 \pm 0$ \\
\hline Quercetin-3-glucoside & $30 \pm 0$ & $30 \pm 0$ & $30 \pm 0$ & $30 \pm 0$ \\
\hline Quercetin-3-galactoside & ND & $60 \pm 0$ & $60 \pm 0$ & $66 \pm 25$ \\
\hline Cyanidin-3-O-glucoside & ND & $30 \pm 0$ & $30 \pm 0$ & $30 \pm 0$ \\
\hline Pelargonidin-3-sambubioside & ND & $102 \pm 16$ & $102 \pm 16$ & $120 \pm 0$ \\
\hline$(+)$ catechin & ND & $30 \pm 0^{3}$ & $42 \pm 16$ & $42 \pm 16$ \\
\hline Pelargonidin-3-O-glucoside & ND & $30 \pm 0$ & $30 \pm 0$ & $30 \pm 0$ \\
\hline Fisetin & ND & $108 \pm 16$ & $90 \pm 0$ & $90 \pm 0$ \\
\hline Pelargonidin-sulfate & ND & $60 \pm 0$ & $60 \pm 0$ & $30 \pm 0$ \\
\hline Quercetin-rutinoside & ND & $240 \pm 0$ & $192 \pm 27$ & $132 \pm 27$ \\
\hline Quercetin & $30 \pm 0^{2}$ & $30 \pm 0^{3}$ & $30 \pm 0$ & $30 \pm 0$ \\
\hline Pelargonidin-3-(6" -malonylglucoside) & ND & $54 \pm 13$ & $30 \pm 0$ & $30 \pm 0$ \\
\hline Pelargonidin-3-O-6"-rhamnosylglucoside & ND & $120 \pm 0$ & $120 \pm 0$ & $114 \pm 13$ \\
\hline Quercetin-glucuronide & ND & $120 \pm 0$ & $120 \pm 0$ & $156 \pm 33$ \\
\hline Quercetin-sulfate & ND & $120 \pm 0$ & $90 \pm 0$ & $90 \pm 0$ \\
\hline 5-Carboxypyranopelargonidin-3-O-beta-glucopyranoside & ND & $180 \pm 0$ & $132 \pm 27$ & $120 \pm 0$ \\
\hline Kaempferol-3-O-sulfate & ND & $120 \pm 0$ & $120 \pm 0$ & $120 \pm 0$ \\
\hline Kaempferol-3-coumaroylglucoside & ND & $36 \pm 13$ & $36 \pm 13$ & $30 \pm 0$ \\
\hline Fisetin-3-rutinoside & ND & $54 \pm 13$ & $42 \pm 16$ & $54 \pm 13$ \\
\hline Methylquercetin & ND & $90 \pm 0$ & $60 \pm 0$ & $60 \pm 0$ \\
\hline Kaempferol-3-(6"-malonylglucoside) & ND & $84 \pm 13$ & $84 \pm 13$ & $60 \pm 0$ \\
\hline Quercetin-3-(6" -caffeoylgalactoside) & ND & $180 \pm 0$ & $180 \pm 0$ & $180 \pm 0$ \\
\hline Kaempferol 3-(6"' -caffeoylglucosyl)-(1->2)-galactoside & ND & $192 \pm 26$ & $168 \pm 27$ & $120 \pm 0$ \\
\hline Quercetin 3-(2",6"-digalloylgalactoside) & ND & $84 \pm 13$ & $60 \pm 0$ & $60 \pm 0$ \\
\hline Pelargonidin3-(6"'-caffeoylglucoside) & ND & $126 \pm 32$ & $108 \pm 16$ & $144 \pm 32$ \\
\hline
\end{tabular}

${ }^{1}$ Values represent Mean time \pm SD based on sampling protocol, $n=5$ subjects, 9 time points per subject. ${ }^{2}$ Detected in two out of five subjects.

${ }^{3}$ Detected in four out of five subjects.

\subsection{LC-MS/MS analysis of pelargonidin glycosides in plasma}

Table 7 shows the maximum concentrations achieved for the most abundant anthocyanins detected in plasma after consuming the four experimental beverages. Maximum concentrations did not significantly differ by dose for pelargonidin-3-O-glucoside; however, concentrations achieved after consumption of the highest dose (40 g strawberry powder-containing beverage) tended to be higher compared to the lowest dose $(10 \mathrm{~g}$ strawberry powder beverage), $p=0.06$. Significant increases were observed across the four doses for the major pelargonidin metabolite, pelargonidin- $O$-glucuronide $(P<0.01)$. Doubling the dose of strawberry powder from $10 \mathrm{~g}$ to $20 \mathrm{~g}$ in the strawberry beverage resulted in $\sim 40 \%$ higher pelargonidin- $O$-glucuronide $\mathrm{C}_{\max }$ and doubling the dose from 


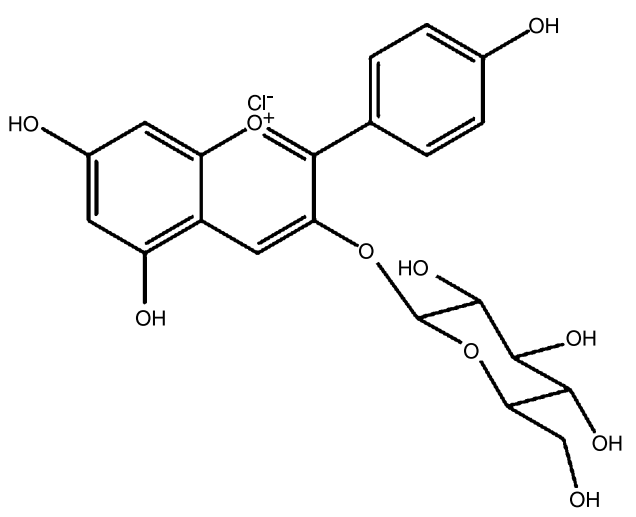

Pelargonidin-3-O-glucoside (1)

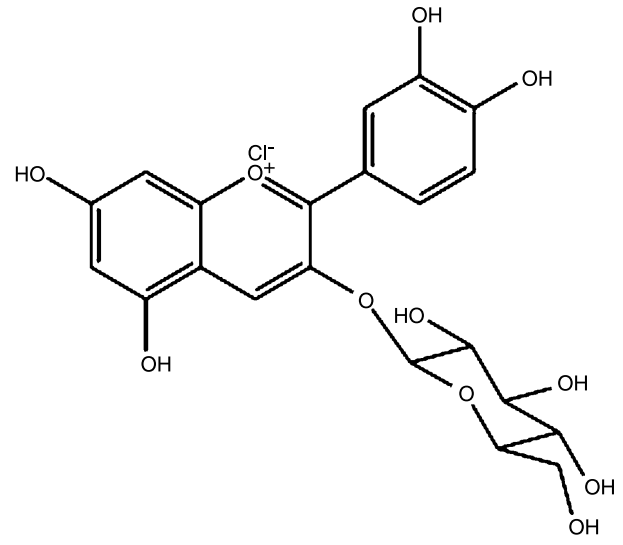

Cyanidin-3-O-glucoside (2)

Fig. 1. Structures of pelargonidin-3-O-glucoside (1) and cyanidin-3-O-glucoside (2), two major anthocyanins present in strawberries.

Table 7

Peak anthocyanin concentrations in plasma ${ }^{1}$

\begin{tabular}{|c|c|c|c|c|}
\hline Compound & $\begin{array}{c}\text { Treatment }{ }^{2} \text { beverage } 0 \mathrm{~g} \\
\text { strawberry powder }\end{array}$ & $\begin{array}{c}\text { Treatment beverage } 10 \mathrm{~g} \\
\text { strawberry powder }\end{array}$ & $\begin{array}{c}\text { Treatment beverage } 20 \mathrm{~g} \\
\text { strawberry powder }\end{array}$ & $\begin{array}{c}\text { Treatment beverage } 40 \mathrm{~g} \\
\text { strawberry powder }\end{array}$ \\
\hline Pelargonidin- $O$-glucuronide $(\mathrm{nmol} / \mathrm{L}$ ) & $0^{\mathrm{a}}$ & $93.4 \pm 21.9^{\mathrm{b}}$ & $166.5 \pm 16.2^{\mathrm{c}}$ & $226 \pm 36.7^{\mathrm{d}}$ \\
\hline Pelargonidin-3-O-glucoside $(\mathrm{nmol} / \mathrm{L})$ & $0^{\mathrm{a}}$ & $10.9 \pm 3.7^{\mathrm{b}}$ & $12.6 \pm 6.5^{\mathrm{b}}$ & $16.4 \pm 9.6^{\mathrm{b} *}$ \\
\hline Cyanidin-3-O-glucoside $(\mathrm{nmol} / \mathrm{L})$ & $0^{\mathrm{a}}$ & $3.8 \pm 0.5^{\mathrm{b}}$ & $4.5 \pm 1.0^{\mathrm{b}}$ & $6.6 \pm 2.7^{\mathrm{c}}$ \\
\hline
\end{tabular}

${ }^{1}$ Values represent Mean $\pm \mathrm{SD}, n=5$. Values with different superscripts in the same row are significantly different each other, $P<0.05 .{ }^{2}$ Treatment beverages (Table 2) containing 0,10, 20, $40 \mathrm{~g}$ freeze-dried strawberry powder. Treatment beverages were consumed with a meal (composition shown in Table 4). *Indicates $P=0.06$ vs $0 \mathrm{~g}$ strawberry powder beverage (placebo).

$20 \mathrm{~g}$ to $40 \mathrm{~g}$ strawberry powder resulted in $\sim 25 \%$ higher pelargonidin- $O$-glucuronide $\mathrm{C}_{\max }$. Peak concentrations were achieved at $148 \pm 31 \mathrm{~min}$. Maximum concentrations achieved after controlling for dose of pelargonidin-3-Oglucoside (Table 8) indicated that pelargonidin- $O$-glucuronide decreased significantly (on a percent dose basis). Pelargonidin- $O$-glucuronide $(\mathrm{m} / \mathrm{z} 447)$ was the most abundant anthocyanin present in plasma after ingestion of strawberry beverages (Fig. 2). Three additional peaks with $\mathrm{m} / \mathrm{z} 447$ and a loss of 176 amu were also detected and corresponded to other isomeric forms of pelargonidin- $O$-glucuronide. Quantitation of pelargonidin- $O$-glucuronide was based only on the most abundant isomer (the additional peaks were under the limit of quantitation (LOQ) level for pelargonidin- $O$-glucuronide).

Figure 3 illustrates the dose-response plasma profile of total pelargonidin- $O$-glucuronide concentrations over $6 \mathrm{~h}$. Consistent with the $\mathrm{C}_{\max }$ data; AUC analysis indicated a significant dose-related increase in pelargonidin- $O$ glucuronide over the $6 \mathrm{~h}$ period (Table 8). However, expressed according to the dose of pelargonidin-3-O-glucoside consumed, AUC of plasma pelargonidin- $O$-glucuronide decreased at the highest dose level ( $40 \mathrm{~g}$ strawberry-powder containing beverage) compared to the $10 \mathrm{~g}$ and $20 \mathrm{~g}$ strawberry powder-containing beverages. The relative percent bioavailability of the parent pelargonidin-3-O-glucoside compound (determined from $6 \mathrm{~h}$ plasma pelargonidin-3-Oglucoside) was $0.26 \% \pm 0.07,0.14 \% \pm 0.05$ and $0.10 \% \pm 0.05$ for 10,20 and $40 \mathrm{~g}$ strawberry powder-containing beverages, respectively. Relative bioavailability of pelargonidin-3-O-glucoside based on its major metabolite pelargonidin- $O$-glucuronide, was $1.7 \% \pm 0.3,1.6 \% \pm 0.3,1.2 \% \pm 0.2$ for $10,20,40 \mathrm{~g}$ strawberry powder-containing beverages, respectively, $p<0.0001)$, consistent with the work of others $[18,12,23]$.

Cyanidin-3- $O$-glucoside is the next most abundant anthocyanin in strawberry $(4.1 \mathrm{mg} / 100 \mathrm{~g} \mathrm{FW})$, which is approximately $6.5 \%$ of total strawberry anthocyanins. The dose of cyanindin-3-O-glucoside consumed in the 4 beverages was $0,3.8 \pm 0.1,9.8 \pm 1.3$ and $22.1 \pm 1.2 \mu \mathrm{mol}$ for $0,10,20,40 \mathrm{mg}$ strawberry powder-containing beverages, respec- 
Table 8

Extent and rate of absorption of pelargonidin-3- $O$-glucoside based on Pelargonidin- $O$-glucuronide in plasma after consumption of placebo and strawberry containing beverages 1

\begin{tabular}{lccccc}
\hline $\begin{array}{l}\text { Treatment } \\
\text { beverages }^{2}\end{array}$ & $\begin{array}{c}\text { Dose }(\mathrm{P} 3 \mathrm{G})^{3} \\
(\mu \mathrm{mol})\end{array}$ & $\begin{array}{c}\mathrm{AUC} \mathrm{PG}^{4} \\
(\mathrm{nmol} / \mathrm{L} / \mathrm{h})\end{array}$ & $\begin{array}{c}\text { AUC PG/Dose } \\
(\mathrm{nmol} / \mathrm{L} / \mathrm{h} / \mu \mathrm{mol})\end{array}$ & $\begin{array}{c}\text { Cmax PG } \\
(\mathrm{nmol} / \mathrm{L})\end{array}$ & $\begin{array}{c}\text { Cmax PG/Dose } \\
(\mathrm{nmol} / \mathrm{L} / \mu \mathrm{mol})\end{array}$ \\
\hline $0 \mathrm{~g}$ & 0 & $0^{\mathrm{a}}$ & $0^{\mathrm{a}}$ & $0^{\mathrm{a}}$ & $0^{\mathrm{a}}$ \\
$10 \mathrm{~g}$ & $81.3 \pm 1.8$ & $315.9 \pm 48.6^{\mathrm{b}}$ & $3.9 \pm 0.6^{\mathrm{b}}$ & $3.3 \pm 21.4^{\mathrm{b}}$ & $1.2 \pm 0.3^{\mathrm{b}}$ \\
$20 \mathrm{~g}$ & $166.8 \pm 4.5$ & $601.2 \pm 68.3^{\mathrm{c}}$ & $3.6 \pm 0.4^{\mathrm{b}}$ & $166.5 \pm 16.2^{\mathrm{c}}$ & $226.7 \pm 36.7^{\mathrm{d}}$ \\
$40 \mathrm{~g}$ & $285.8 \pm 8.9$ & $790.5 \pm 61.2^{\mathrm{d}}$ & $2.8 \pm 0.2^{\mathrm{c}}$ & $0.8 \pm 0.1^{\mathrm{d}}$ \\
\hline
\end{tabular}

${ }^{1}$ Values represent Mean $\pm \mathrm{SD}, n=5$ except for $\mathrm{P} 3 \mathrm{G}$ as noted below $\left({ }^{3}\right)$. Values with different superscripts in the same column are significantly different from each other, $P<0.05 .{ }^{2}$ Treatment beverages are the beverages containing $0,10,20,40 \mathrm{~g}$ freeze-dried strawberry powder (Table 2 ). Treatment beverages were consumed with a meal (Table 4). ${ }^{3} \mathrm{P} 3 \mathrm{G}=$ pelargonidin-3-O-glucoside. Values represent Mean $\pm \mathrm{SD} .{ }^{4} \mathrm{PG}=\mathrm{Pelargonidin}-$ $O$-glucuronide.

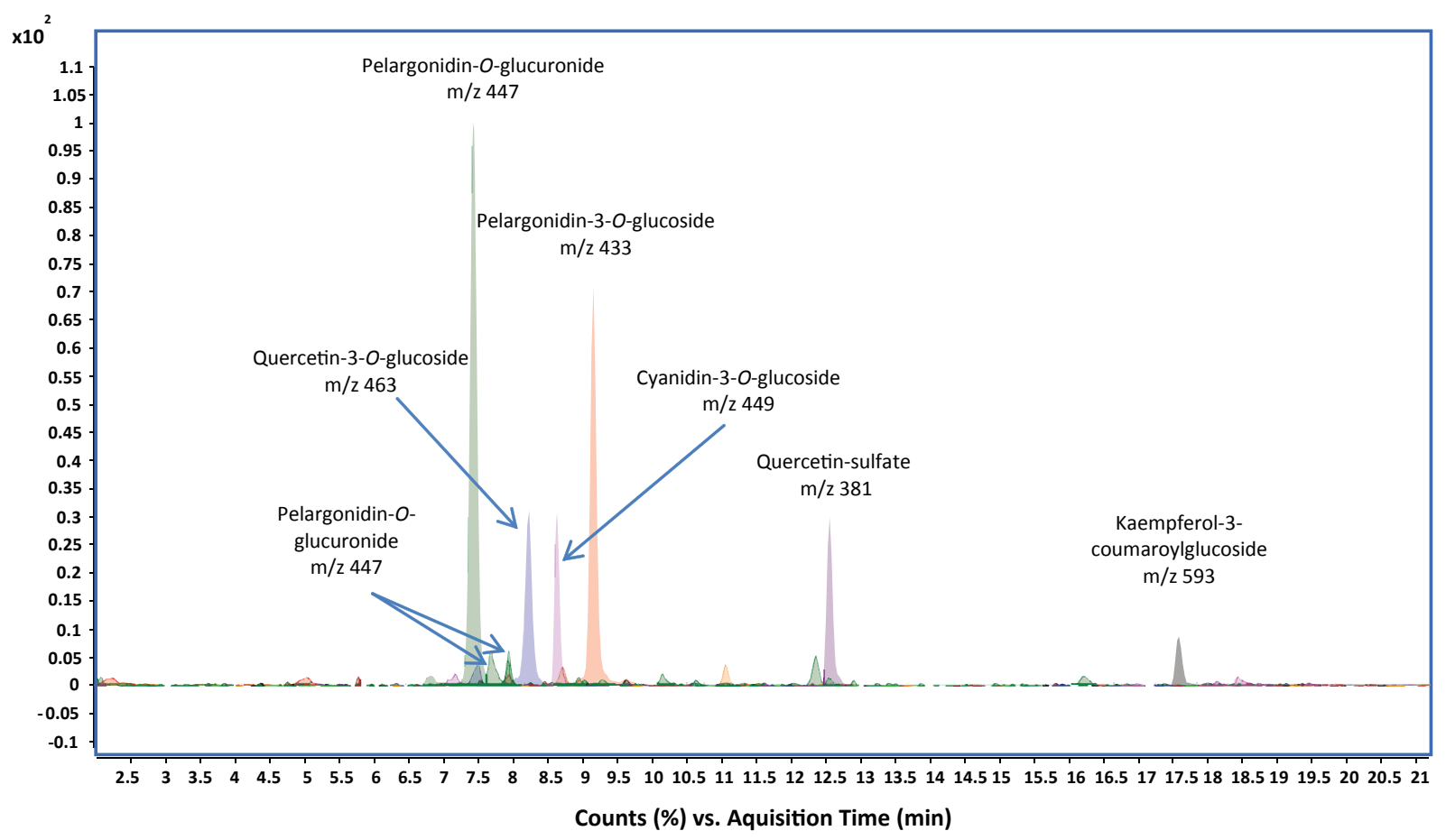

Fig. 2. Representative LC-MS/MS-MRM profile of human plasma after consumption of strawberry beverage containing $40 \mathrm{~g}$ of freeze dried strawberry powder with a meal at 120 min post prandial.

tively. Maximum concentrations of cyanidin-3- $O$-glucoside increased $\sim 20 \%$ after doubling the dose from 10 to $20 \mathrm{mg}$ and $\sim 30 \%$ after doubling the dose from 20 to $40 \mathrm{~g}$. Internal standard (delphinidin-3-O-glucoside) recoveries were in a range of $88-115 \%$ for all analyzed samples.

\section{Discussion}

The purpose of the present study was to contribute to the growing body of knowledge of anthocyanin metabolism focusing on pelargonidin-based anthocyanins and their metabolites in plasma after consuming multiple doses of strawberry in a beverage with a complete meal using targeted and non-targeted analysis approaches enabled by 


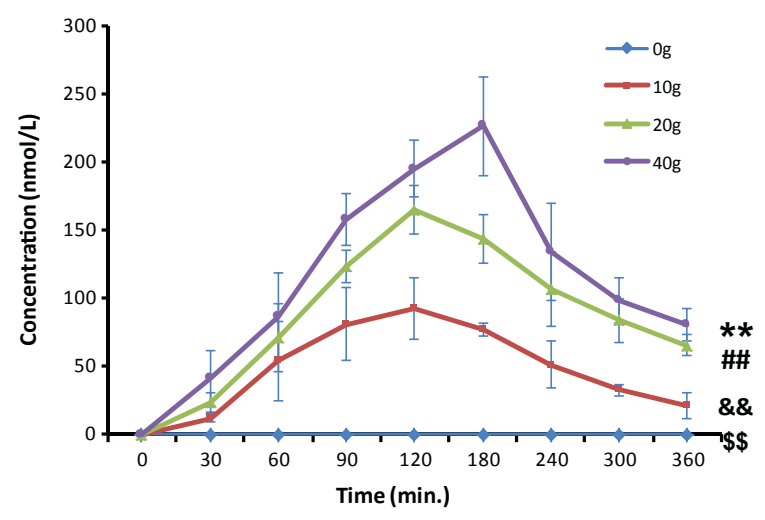

Fig. 3. Analysis of pelargonidin- $O$-glucuronide in plasma samples in response to strawberry beverages containing $0,10,20,40 \mathrm{~g}$ freeze-dried strawberry powder using LC-MS/MS. Values represent the Mean \pm SEM of pelargonidin- $O$-glucuronide concentrations at respective time points

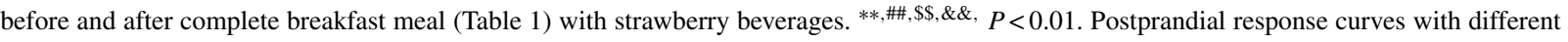
symbols are significantly different from each other as estimated by area under the curve, AUC $(n=5)$.

advances in mass spectrometry. The findings of this study confirm previous work that pelargonidin- $O$-glucuronide is the primary pelargonidin metabolite found in plasma after strawberry consumption $[11,15,18,21,25,26]$. However, while the maximum concentration in plasma and area under the concentration-time curve increased with dose; concentrations of pelargonidin- $O$-glucuronide decreased as a percent of dose, suggesting possible saturation of absorptive mechanisms or increased efficiency in elimination. The non-targeted Q-TOF LC/MS analysis was consistent with the requisite for identification of new and confirmation of previously reported metabolites in postprandial plasma after strawberry consumption. Furthermore, the analysis presented novel insights into the timeframe of metabolic changes that occur after consumption of pelargonidin-rich strawberry containing beverages.

The emerging health benefits of anthocyanins and strawberries as a favorite fruit to deliver anthocyanins warrants investigation of the absorption and metabolism of strawberry anthocyanins. To our knowledge, only 2 other investigations have examined bioavailability of strawberry anthocyanins over multiple doses [22, 23] and both utilized urinary excretion as a means for estimating bioavailability. Carkeet et al. [23] reported that strawberry anthocyanin (from puree) excretion increases linearly with doses from 15-60 $\mu \mathrm{mol}$ anthocyanins and recovery as a percent of dose was $\sim 2 \%$. Hollands et al. [22] reported that doses of fresh strawberries (100-400 g) delivering approximately 57-228 $\mu \mathrm{mol}$ anthocyanins per dose also resulted in linear urinary anthocyanin excretion. In the present study, a dose range of 101-368 $\mu \mathrm{mol}$ strawberry anthocyanins $(\sim 81-286 \mu \mathrm{mol}$ pelargonidin-3-O-glucoside) was ingested and plasma concentrations of major anthocyanins and their metabolites were quantified. Direct measurement of the maximum concentrations achieved $\left(\mathrm{C}_{\max }\right)$ and calculations of extent of exposure $\left(\mathrm{AUC}_{0-6 \mathrm{~h}}\right)$ indicated dose-related increases in both kinetic parameters during the $6 \mathrm{~h}$ experimental period. However, after controlling for dose, $\mathrm{AUC}_{0-6 \mathrm{~h}}$ decreased $\sim 28 \%$ at the highest strawberry dose compared to the $10 \mathrm{~g}$ dose. Decreases of $\sim 17$ and $20 \%$ were also apparent relative to dose based on $\mathrm{C}_{\max }$ comparing differences from 10 to $20 \mathrm{~g}$ strawberry powder in the beverages and 20 to $40 \mathrm{~g}$ strawberry powder in the beverages, respectively. These decreases suggest saturation of absorptive mechanisms or increased rates of elimination. We found no significant differences in elimination rate constants $\left(\mathrm{k}_{\mathrm{e}}\right)$ among doses: 10, 20, $40 \mathrm{~g}$ strawberry powder containing beverages, $\mathrm{k}_{\mathrm{e}}=0.09 \pm 0.02 \mathrm{~h}^{-1}, 0.10 \pm 0.02 \mathrm{~h}^{-1}, 0.12 \pm 0.04 \mathrm{~h}^{-1}$, respectively, suggesting but not confirming changes in efficiency at the level of absorption. Kurilich et al., reported maximal absorption at $350 \mu \mathrm{mol}$ (or less) of cyanidin-based anthocyanins, and saturation mechanisms for tea catechins and carotenoids has also been reported [19, 28, 29]. The apparent saturation supports absorptive transporter involvement, which has been described and reviewed previously [30]. It is noteworthy that our experimental design like others is based on single doses of strawberry. Ferruzzi and colleagues (2009) reported increased bioavailability of gallic acid and catechins from higher doses of grape seed polyphenol extract with repeated dosing in rats compared to a single dose protocol [31]. Whether regular consumption of higher doses of strawberries (anthocyanins) would increase efficiency of absorption of these compounds requires further investigation. 
Previously published human studies investigating strawberry anthocyanins kinetic parameters have tested anthocyanin absorption and excretion after ingestion of a single dose in various forms (fresh or frozen strawberries) with little or no accompanying food [18, 21-23, 32]. Administering anthocyanin-rich fruit with or without food/meal, as well as composition of the food/meal would be expected to affect absorption and or the kinetics of valuable bioactives, as suggested by Mullen et al. [21]. In this study, subjects consumed four different doses of strawberry in a beverage with a meal representing modern day western eating patterns. Modern day meals induce oxidative stress and inflammation due to their relatively high and readily available carbohydrate and fat content. One of the beneficial effects anthocyanin-rich fruits may have on health is to relieve meal-induced oxidative and metabolic imbalances. Therefore, strawberry anthocyanin/pelargonidin bioavailability was assessed and polyphenols and metabolites were profiled during a typical post meal period. Estimates of bioavailability from other studies ranged from $0.75 \%$ to $2.4 \%[18,21-23]$ and in the present study we estimated bioavailability to range from $1.2-1.7 \%$, decreasing at the highest dose of strawberry consumed (40 g strawberry powder, $\sim 286 \mu \mathrm{mol}$ pelargonidin-3-O-glucoside). Our estimates of bioavailability in general $(\sim 1.5 \%)$ are consistent with previous reports. This is the first time that we are aware strawberry anthocyanin bioavailability has been examined in a large meal setting, which could have played a role in anthocyanin bioavailability. One limitation of the study was that we only had plasma collections to $6 \mathrm{~h}$ postprandial. Because of the large meal, absorption would be expectedly slowed (compared to drinking the beverages alone) resulting in prolonged absorption time and potentially increased absorption at lower segments of the small intestine. Likewise, metabolite contribution from large bowel microbial metabolism may be altered depending on remaining substrate to pass from the small to large bowel. Whether the metabolite profile would change under these conditions is speculative and would not be reflected in the glucuronide metabolites we focused on in this study for assessing bioavailability. Assessing time of appearance of various plasma compounds provided insight into the profile of metabolites within different time frames postprandially according to dose; however, a second limitation of the study was that we did not have a second control arm whereby beverages were consumed without a meal. Nonetheless, the time of appearance analysis provided another example of how chemical structure of polyphenols modulates their intestinal absorption. The rate and extent of metabolite appearance in plasma varies from one phenolic compound to the next and can also be affected by the foods it is consumed with [21]. Chemical structures of dietary polyphenols also influence their conjugation with glucuronide, sulfate or methyl groups and the amounts of formed metabolites [33]. The conjugates can be also hydrolyzed by the colonic microbiota and the resulting aglycone reabsorbed or further metabolized to low molecular weight aromatic acids such as, observed in current study, 4-hydroxybenzoic acid. Thus, understanding the structural features affecting absorption of polyphenolic compounds is a key in determining their metabolic path. Future studies will need to be longer in time and have a control condition without a meal to discern the contribution of alimentary handling on anthocyanins/pelargondinin absorption and metabolism.

Anthocyanins and their metabolites were identified in plasma using LC-MS/MS and Q-TOF LC/MS as described in the methods. Numerous compounds were identified, of which pelargonidin- $O$-glucuronide, pelargonidin-3- $O$ glucoside and cyanidin-3-O-glucoside were the most abundant anthocyanins. However, identification of metabolites of polyphenolic compounds is challenging due to their complexity and limited database information. Metabolized polyphenolic compounds could be transformed into structures not detected by currently available analytical methods. Discrepancy in available literature also makes it difficult to establish methods for analyzing anthocyanin derived metabolites, but these data were confirmed using Q-TOF LC/MS. This technology enables automatic acquisition of MS spectra and MS/MS in a single chromatographic injection. The Q-TOF analysis allows to obtain the exact mass of ions, which is essential for the assignment of elemental composition and therefore for characterization of small molecules. While Q-TOF LC/MS does not provide the ultimate detection limits compared to advanced triple-quadruple systems, its sensitivity is sufficient for compound assessment in bioavailability studies. It enables identification, profiling and structure elucidation of metabolites by producing high quality MS and MS/MS spectra with accurate isotope ratios. Combined with sophisticated data analysis software and databases, Q-TOF mass spectrometry provides a clear advantage over other technologies in non-targeted analysis of bioactives. The data extracted from Q-TOF LC/MS analysis allowed for detection of 7 previously undescribed compounds in plasma and also allowed for metabolite profiling based on time of first appearance. These data provide insight into the array of compounds that are generated, a sense of the time course of appearance of various compounds and a snapshot of variability among subjects (several were detected for the first time at the same time point and only 3 compounds were detected in less than the total sample set). Review articles tabulating the major compounds and metabolites detected 
after fruit consumption with their general kinetic profile $\left(\mathrm{C}_{\max }, \mathrm{T}_{\max }\right)$ have been published; [34, 35] however, an extensive qualitative profiling as shown in the present study has not been documented.

The Q-TOF technology, utilized also by Hanhineva in two studies on strawberry leaves and flowers [36, 37], enables characterization of compounds based not only on accurate mass but also on fragmentation pattern of the parent ion, increasing the confidence in compound identification. Therefore, it was important to utilize the accuracy of nontargeted Q-TOF analysis in determining additional polyphenolic metabolites (Table 7) and times of their appearance in plasma (Table 8) relevant to possible physiological effects on inflammation, oxidative stress and insulin resistance. Enriching the knowledge of postprandial plasma metabolites and their appearance over time can provide insight into further understanding of the impact of colonic microbiota on absorption and metabolism of dietary polyphenols and allow better perception of biological effect of the polyphenolic metabolites. The accuracy of mass data generated by Q-TOF LC/MS together with the fragmentation pattern of the full scan run of MS/MS analysis have been a useful tool to tentatively characterize phenolic compounds in the plasma matrix. Most of these compounds found in human plasma are being reported here for the first time. Full-spectrum acquisition at good sensitivity with Q-TOF analysis allowed detection and characterization of compounds, such as fisetin and its glycoside, not identified in the strawberry beverages analyzed by targeted LC-MS/MS. Nonetheless, both targeted and non-targeted approaches taken in this study are complementary and both have their advantages in determination of plasma metabolites.

\section{Acknowledgments}

The present study was funded by a grant from the California Strawberry Commission and California Department of Food and Agriculture. Chemical analysis of strawberry was conducted at the Institute for Food Safety and Health (Bedford Park, IL, USA) under the corporative agreement grant with the Food and Drug Administration. Agilent Technologies, USA provided instrumentation and necessary technical advices. The authors would like to acknowledge the contributions of Julie Talbot MS RD, Sarah Thomas MS RD, Lourdes Alandete, Lakshmi Vijayakumar and Hequn Wei for coordinating the trial. The authors do not have any conflicts of interest with any commercial sponsor. B. M. B-F. and I.E designed the study; K.B., E.P., J.C., I. E. and B.M.B-F. conducted the study; B.M. B-F., I.E. and K.B. analyzed the data and compiled the manuscript. All authors read and approved the manuscript.

\section{References}

[1] Aaby K, Ekeberg D, Skrede G. Characterization of phenolic compounds in strawberry (Fragaria x ananassa) fruits by different HPLC detectors and contribution of individual compounds to total antioxidant capacity. J Agric Food Chem 2007;55(11):4395-406.

[2] Seeram NP, Lee R, Scheuller S, Heber D. Identification of phenolic compounds in strawberries by liquid chromatography electrospray ionization mass spectroscopy. Food Chem 2006;(97):1-11.

[3] Aaby K, Skrede G, Wrolstad RE. Phenolic composition and antioxidant activities in flesh and achenes of strawberries (Fragaria ananassa). J Agric Food Chem 2005;53(10):4032-40.

[4] Prasain JK, Barnes S. Metabolism and bioavailability of flavonoids in chemoprevention: Current analytical strategies and future prospectus. Mol Pharm 2007;4(6):846-64.

[5] Cerdá B, Tomás-Barberán FA, Espín JC. Metabolism of antioxidant and chemopreventive ellagitannins from strawberries, raspberries, walnuts, and oak-aged wine in humans: Identification of biomarkers and individual variability. J Agric Food Chem 2005;53(2):227-35.

[6] Basu A, Fu DX, Wilkinson M, Simmons B, Wu M, Betts NM, et al. Strawberries decrease atherosclerotic markers in subjects with metabolic syndrome. Nutr Res 2010;30(7):462-9.

[7] Pinto MaS, de Carvalho JE, Lajolo FM, Genovese MI, Shetty K. Evaluation of antiproliferative, anti-type 2 diabetes, and antihypertension potentials of ellagitannins from strawberries (Fragaria $\times$ ananassa Duch.) using in vitro models. J Med Food 2010;13(5):1027-35.

[8] Törrönen R, Sarkkinen E, Tapola N, Hautaniemi E, Kilpi K, Niskanen L. Berries modify the postprandial plasma glucose response to sucrose in healthy subjects. Br J Nutr 2010;103(8):1094-7.

[9] Maher P, Dargusch R, Ehren JL, Okada S, Sharma K, Schubert D. Fisetin Lowers Methylglyoxal Dependent Protein Glycation and Limits the Complications of Diabetes. PLoS ONE 2011;6(6):e21226.

[10] Joseph JA, Shukitt-Hale B, Casadesus G. Reversing the deleterious effects of aging on neuronal communication and behavior: Beneficial properties of fruit polyphenolic compounds. Am J Clin Nutr 2005;81(1 Suppl):313S-6S. 
[11] Henning SM, Seeram NP, Zhang Y, Li L, Gao K, Lee RP, et al. Strawberry consumption is associated with increased antioxidant capacity in serum. J Med Food 2010;13(1):116-22.

[12] Basu A, Wilkinson M, Penugonda K, Simmons B, Betts NM, Lyons TJ. Freeze-dried strawberry powder improves lipid profile and lipid peroxidation in women with metabolic syndrome: Baseline and post intervention effects. Nutr J 2009;8:43.

[13] Burton-Freeman B. Postprandial metabolic events and fruit-derived phenolics: A review of the science. Br J Nutr 2010;104(Suppl 3):S1-14.

[14] Jenkins DJ, Nguyen TH, Kendall CW, Faulkner DA, Bashyam B, Kim IJ, et al. The effect of strawberries in a cholesterol-lowering dietary portfolio. Metabolism 2008;57(12):1636-44.

[15] Edirisinghe I, Banaszewski K, Cappozzo J, Sandhya K, Ellis CL, Tadapaneni R, et al. Strawberry anthocyanin and its association with postprandial inflammation and insulin. Br J Nutr 2011;106:913-22.

[16] Ellis CL, Edirisinghe I, Kappagoda T, Burton-Freeman B. Attenuation of meal-induced inflammatory and thrombotic responses in overweight men and women after 6-week daily strawberry (Fragaria) intake. A randomized placebo-controlled trial. J Atheroscler Thromb 2011;18:318-27.

[17] McGhie TK, Walton MC. The bioavailability and absorption of anthocyanins: Towards a better understanding. Mol Nutr Food Res 2007;51(6):702-13.

[18] Azzini E, Vitaglione P, Intorre F, Napolitano A, Durazzo A, Foddai MS, et al. Bioavailability of strawberry antioxidants in human subjects Br J Nutr 2010;104(8):1165-73.

[19] Kurilich AC, Clevidence BA, Britz SJ, Simon PW, Novotny JA. Plasma and urine responses are lower for acylated vs nonacylated anthocyanins from raw and cooked purple carrots. J Agric Food Chem 2005;53(16):6537-42.

[20] Charron CS, Kurilich AC, Clevidence BA, Simon PW, Harrison DJ, Britz SJ, et al. Bioavailability of anthocyanins from purple carrot juice: Effects of acylation and plant matrix. J Agric Food Chem 2009;57(4):1226-30.

[21] Mullen W, Edwards CA, Serafini M, Crozier A. Bioavailability of pelargonidin-3-O-glucoside and its metabolites in humans following the ingestion of strawberries with and without cream. J Agric Food Chem 2008;56(3):713-9.

[22] Hollands W, Brett GM, Dainty JR, Teucher B, Kroon PA. Urinary excretion of strawberry anthocyanins is dose dependent for physiological oral doses of fresh fruit. Mol Nutr Food Res 2008;52(10):1097-105.

[23] Carkeet C, Clevidence BA, Novotny JA. Anthocyanin excretion by humans increases linearly with increasing strawberry dose. J Nutr 2008;138(5):897-902.

[24] Roback JD, Grossman BJ, Harris T, Hillyer CD. Technical Bulletin of American Association of Blood Banks (AABB). 2011.

[25] Mullen W, Larcombe S, Arnold K, Welchman H, Crozier A. Use of accurate mass full scan mass spectrometry for the analysis of anthocyanins in berries and berry-fed tissues. J Agric Food Chem 2010;58(7):3910-5.

[26] Hanhineva K, Kärenlampi S, Aharoni A. Genes, Genomes and Genomics. UK: Global Science Books, Ltd; 2010. pp. 65-75.

[27] Maher P, Akaishi T, Abe K. Flavonoid fisetin promotes ERK-dependent long-term potentiation and enhances memory. Proc Natl Acad Sci U S A 2006;103(44):16568-73.

[28] Yang CS, Chen L, Lee MJ, Balentine D, Kuo MC, Schantz SP. Blood and urine levels of tea catechins after ingestion of different amounts of green tea by human volunteers. Cancer Epidemiol Biomarkers Prev 1998;7(4):351-4.

[29] Diwadkar-Navsariwala V, Novotny JA, Gustin DM, Sosman JA, Rodvold KA, Crowell JA, et al. A physiological pharmacokinetic model describing the disposition of lycopene in healthy men. J Lipid Res 2003;44(10):1927-39.

[30] Wu X, Cao G, Prior RL. Absorption and metabolism of anthocyanins in elderly women after consumption of elderberry or blueberry. J Nutr 2002;132(7):1865-71.

[31] Ferruzzi MG, Lobo JK, Janle EM, Cooper B, Simon JE, Wu QL, et al. Bioavailability of gallic acid and catechins from grape seed polyphenol extract is improved by repeated dosing in rats: Implications for treatment in Alzheimer's disease. J Alzheimers Dis 2009;18(1):113-24.

[32] Felgines C, Talavéra S, Gonthier MP, Texier O, Scalbert A, Lamaison JL, et al. Strawberry anthocyanins are recovered in urine as glucuroand sulfoconjugates in humans. J Nutr 2003;133(5):1296-301.

[33] Giampieri F, Tulipani S, Alvarez-Suarez J, Quiles J, Mezzetti B, Battino M. The strawberry: Composition, nutritional quality and impact on human health. Nutrition 2012;28(1):9-19.

[34] Crozier A, Jaganath IB, Clifford MN. Dietary phenolics: Chemistry, bioavailability and effects on health. Nat Prod Rep 2009;26(8):1001-43.

[35] Manach C, Williamson G, Morand C, Scalbert A, Rémésy C. Bioavailability and bioefficacy of polyphenols in humans. I. Review of 97 bioavailability studies. Am J Clin Nutr 2005;81(1 Suppl):230S-42S.

[36] Hanhineva K, Soininen P, Anttonen MJ, Kokko H, Rogachev I, Aharoni A, et al. NMR and UPLC-qTOF-MS/MS characterisation of novel phenylethanol derivatives of phenylpropanoid glucosides from the leaves of strawberry (Fragaria $\mathrm{x}$ ananassa cv. Jonsok). Phytochem Anal 2009;20(5):353-64.

[37] Hanhineva K, Rogachev I, Kokko H, Mintz-Oron S, Venger I, Kärenlampi S, et al. Non-targeted analysis of spatial metabolite composition in strawberry (Fragariaxananassa) flowers. Phytochemistry 2008;69(13):2463-81. 